\title{
Investigation of Symmetries of Second-Order Nonlinear Susceptibility Tensor Based on THz Generation
}

\author{
Guibao Xu, Guan Sun, and Yujie J. Ding \\ Department of Electrical and Computer Engineering, Lehigh University, Bethlehem, PA 18015 \\ yud2@lehigh.edu \\ Ioulia B. Zotova \\ ArkLight, P.O. Box 2, Center Valley, PA 18034 \\ Krishna C. Mandal, Alket Mertiri, and Gary Pabst \\ EIC Laboratories, Inc., Norwood, MA 02062 \\ Nils Fernelius \\ Materials and Manufacturing Directorate, Air Force Research Laboratory, Wright-Patterson AFB, OH 45433
}

\begin{abstract}
We demonstrate that $\mathrm{THz}$ generation can be a sensitive technique for investigating symmetries of second-order nonlinear susceptibility tensor. (C)2010 Optical Society of America

OCIS codes: (190.4720) Optical nonlinearities of condensed matter; (190.4400) Nonlinear optics, materials
\end{abstract}

\section{Introduction}

For second-order nonlinear susceptibility, Kleinman symmetry condition must be satisfied when all the frequencies of the waves participating in the parametric interaction are much lower than the lowest resonance frequency of a nonlinear material. In such a case, one can permute the indices of the elements for the second-order nonlinear susceptibility tensor without permuting the frequencies. Furthermore, spatial symmetry of a nonlinear material imposes additional relations among some of the elements of the second-order nonlinear susceptibility tensor.

Here, we demonstrate that $\mathrm{THz}$ generation can be a sensitive technique for investigating symmetries of secondorder nonlinear susceptibility tensor. Indeed, using GaSe as an example, our result illustrates that both Kleinman and spatial symmetries are violated for $\mathrm{THz}$ generation, which can be attributed to the contribution of the slightly-lossy frequency-dependent ionic second-order nonlinear susceptibility of the material.

If Kleinman and spatial symmetry conditions are satisfied, the elements for the second-order nonlinear susceptibility tensor for GaSe satisfy the relations of $d_{16}=d_{21}$ and $d_{22}=-d_{21}$. When the THz output frequency is close to the transverse-optical (TO) phonon frequency of $6.41 \mathrm{THz}(46.8 \mu \mathrm{m})$ for GaSe [1], GaSe becomes lossy, and therefore, the ionic second-order nonlinear coefficients are frequency dependent. In the past, GaSe was used to generate $\mathrm{THz}$ pulses from ultrafast laser pulses with the output power reaching $5.4 \mu \mathrm{W}$ [2].

\section{GaSe crystals and experiment}

GaSe crystals in our experiment were grown by a Bridgman method. They were doped by $\mathrm{In}$ and $\mathrm{Cr}$ in order to improve their hardness. They have a typical dimension of $13 \times 8 \times 1 \mathrm{~mm}^{3}$ with the two facets perpendicular to the $c$ axis being separated by $1 \mathrm{~mm}$. Broadband THz pulses were generated by using a Ti:sapphire regenerative amplifier at the wavelength of $782 \mathrm{~nm}$ and a coherent beam at $391 \mathrm{~nm}$ achieved through frequency-doubling in a BBO crystal. The pulse duration and repetition rate for the two output beams are $180 \mathrm{fs}$ and $250 \mathrm{kHz}$, respectively. When the excitation beam was focused onto one of the crystal facets, the THz radiation was collimated and then focused to a power meter for measuring the $\mathrm{THz}$ output powers in the transmission and reflection geometries.

Table 1. Output powers measured on GaSe crystals.

\begin{tabular}{cccc}
\hline \multirow{2}{*}{ Crystal ID } & Dopant (ppm) & \multicolumn{2}{c}{ Output Power } \\
\cline { 3 - 4 } & & $\mu \mathbf{W}$ & $\mathbf{n W}$ \\
\hline GSU7903 & undoped & 19.41 & 60 \\
GSI8701 & In (500) & 22.93 & 37 \\
GSI7801 & In (3000) & 16.33 & 43 \\
GSC8902 & Cr (500) & 19.45 & 37 \\
\hline
\end{tabular}

\section{Results and discussions}

At the average pump power of $1.14 \mathrm{~W}$ at $782 \mathrm{~nm}$, the highest $\mathrm{THz}$ output powers were measured to be in the range of 16.3-22.9 $\mu \mathrm{W}$ from four GaSe crystals in the transmission geometry, see Table 1. The highest output power 
among different doped crystals was measured to be $22.9 \mu \mathrm{W}$ from GSI8701, which is a factor of 4.2 higher than our previous result [2]. It appears to us that the second-order nonlinear coefficient can be increased by introducing a small amount of In. This is consistent with one of the previous reports [3]. However, based on our result, introducing $\mathrm{Cr}$ at the level of $500 \mathrm{ppm}$ did not affect the second-order nonlinear coefficient. According to Fig. 1, the power dependence is close to a quadratic dependence. Above $618 \mathrm{~W} / \mathrm{cm}^{2}$, the deviation of our data from the quadratic dependence is caused by two-photon absorption of GaSe at $782 \mathrm{~nm}$ [4]. We have attributed the mechanism of the $\mathrm{THz}$ generation in these four GaSe crystals to optical rectification.
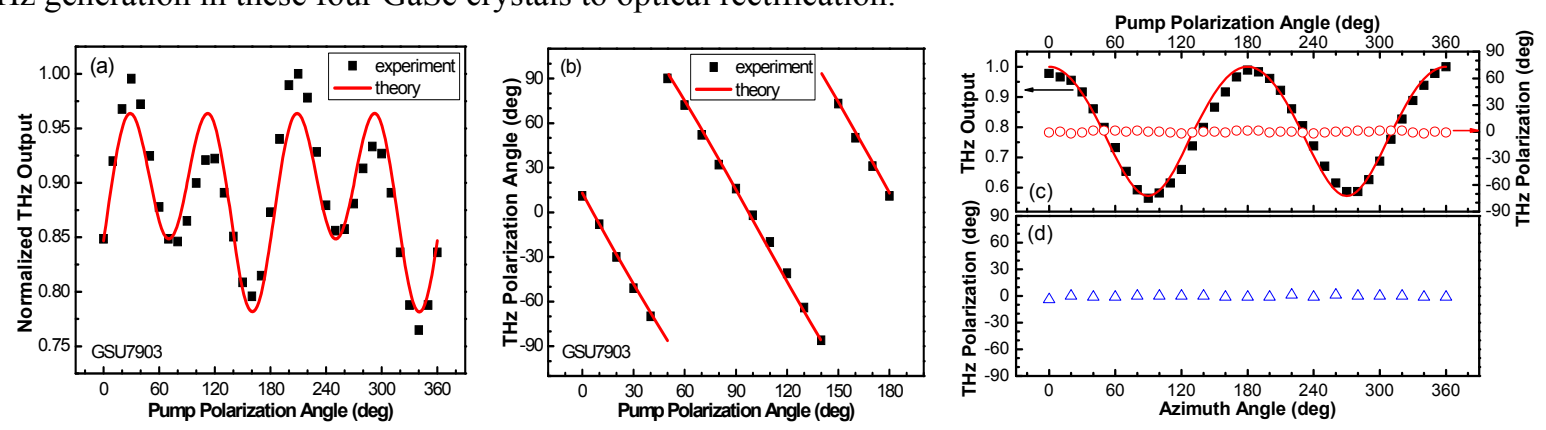

Fig. 2. (a) THz output power vs. pump polarization angle at $782 \mathrm{~nm}$, (b) $\mathrm{THz}$ polarization vs. pump polarization angle at $782 \mathrm{~nm}$, (c) THz output power and polarization vs. azimuth angle at $391 \mathrm{~nm}$, and (d) THz polarization vs. azimuth angle at $391 \mathrm{~nm}$. Squares, circles, and triangles correspond to data; solid curves correspond to fitting to the data points.

At normal incidence, THz nonlinear polarizations are given by $P_{x}=4 d_{16} E_{x} E_{y}$ and $P_{y}=2 d_{21} E_{x}{ }^{2}+2 d_{22} E_{y}{ }^{2}$. Using $E_{x}$ $=E_{0} \cos \theta$ and $E_{y}=E_{0} \sin \theta$, where $\theta$ is the pump polarization angle, the THz intensity and polarization angle are

$$
I_{T} \propto 2\left(\frac{d_{16}}{d_{22}}\right)^{2} \sin ^{2}(2 \theta)+\left(\frac{d_{21}}{d_{22}}\right) \sin ^{2}(2 \theta)+2\left(\frac{d_{21}}{d_{22}}\right)^{2} \cos ^{4} \theta+2 \sin ^{4} \theta, \theta_{T}=\tan ^{-1}\left[\frac{\left(d_{21} / d_{22}\right) \cos ^{2} \theta+\sin ^{2} \theta}{\left(d_{16} / d_{22}\right) \sin (2 \theta)}\right]
$$

By adjusting $d_{16} / d_{22}$ and $d_{21} / d_{22}$, we can use Eq. (1) to achieve the nonlinear least square fit to the data presented in Fig. 2(a). As a result, we have obtained $d_{16} \approx 0.979 d_{21}$ and $d_{22} \approx-1.04 d_{21}$. In comparison, if $d_{16}=d_{21}$ and $d_{22}=-d_{21}$, the THz output power would be independent of the pump polarization angle. Therefore, even when $d_{16}$ and $d_{22}$ deviate from $d_{21}$ and $-d_{21}$ by as small as $2 \%$ and $4 \%$, respectively, the THz output power exhibits a measurable oscillation as a function of the pump polarization angle. Based on our result, we have demonstrated that such an oscillation characteristic can be used to determine $d_{16} / d_{21}$ and $d_{22} / d_{21}$ for GaSe crystals. We believe that such small deviations originate from the low loss for the THz wave. Indeed, a THz wave can be coupled with TO phonons in $\mathrm{GaSe}$, resulting in the absorption of the $\mathrm{THz}$ wave [5]. Since the peak output wavelength of $300 \mu \mathrm{m}$ is much longer than the corresponding wavelength for the TO phonons, the THz output wave suffers from low loss. Consequently, due to the slightly-lossy ionic second-order nonlinear susceptibility contributing to the THz generation, Kleinman symmetry condition is no longer satisfied. On the other hand, due to asymmetric residual strain following the crystal growth and cleavage, spatial symmetry is violated. Despite of the oscillation observed in Fig. 2(a), the dependence of the THz polarization angle on the pump polarization angle is close to linear, see Fig. 2(b).

When we used the wavelength of $391 \mathrm{~nm}$ to pump the GaSe crystals, the THz output powers measured under the reflection geometry are 2-3 orders of magnitude lower, see Table 1 . At an incident angle of $71^{\circ}$ for the pump beam, the polarization angle for the $\mathrm{THz}$ beam is kept as a constant regardless of the pump polarization angle or azimuth angle, see Figs. 2(c) and 2(d). Such a behavior is a clear indication that the mechanism for the THz generation pumped at $391 \mathrm{~nm}$ is photocurrent surge. THz output power vs. polarization angle exhibits two-period oscillation within $0-360^{\circ}$. This is completely different from the $\mathrm{THz}$ characteristic due to optical rectification. After including the Fresnel loss of the pump beam, our data in Fig. 2(c) can be well fitted by our theory based on photocurrent surge.

This work has been supported by U.S. Air Force Research Laboratory.

\section{References}

[1] N. Piccioli, R. Le Toullec, M. Mejatty, and M. Balkanski, "Refractive index of GaSe between $0.45 \mu \mathrm{m}$ and $330 \mu \mathrm{m}$," Appl. Opt. 16, 12361238 (1977).

[2] X. Mu, I. B. Zotova, and Y. J. Ding, "Power scaling on efficient generation of ultrafast terahertz pulses," IEEE J. OF Sel. Top. Quantum Electron. 14, 315-332 (2008).

[3] D. R. Suhre, N. B. Singh, V. Balakrishna, N. C. Fernelius, and F. K. Hopkins, Opt. Lett. 22, 775 (1997).

[4] I. B. Zotova and Y. J. Ding, "Spectral measurements of two-photon absorption coefficients for CdSe and GaSe crystals," Appl. Opt. 40, 6654$6658(2001)$.

[5] T. Dekorsy, V. A. Yakovlev, W. Seidel, M. Helm, and F. Keilmann, "Infrared-phonon-polariton resonance of the nonlinear susceptibility in GaAs," Phys. Rev. Lett. 90, 055508/1-4 (2003). 